\title{
Kondo Lattice Models for Rare-Earth and Actinide Systems
}

\author{
B. COQBLin*
}

\author{
Laboratoire de Physique des Solides, UMR 8502, CNRS — Université Paris-Sud, 91405-Orsay, France
}

\begin{abstract}
We present here some works on the strong competition between the Kondo effect, magnetic order and eventually spin glass or frustration effect in anomalous rare-earth and actinide systems. First, we develop an underscreened Kondo lattice model with $S_{f}=1 \mathrm{spins}$ for the $5 f$-electrons and we have recently improved it by deriving, by the Schrieffer-Wolff transformation, a $5 f$-band with a finite bandwidth. The underscreened Kondo lattice model can account for properties of some uranium and neptunium compounds, like UTe, $\mathrm{Np}_{2} \mathrm{PdGa}_{3}$ or $\mathrm{UCu}_{2} \mathrm{Si}_{2}$ which have a large Curie temperature $T_{\mathrm{c}}$ of order $100 \mathrm{~K}$ and present also a Kondo behavior. In particular, we can account for the observed maximum of $T_{\mathrm{c}}$ under pressure in UTe and the magnetization curves of $\mathrm{NpNiSi}_{2}$ showing the occurrence of the Kondo effect at low temperatures below $T_{\mathrm{c}}$. Second, we have studied the properties of disordered cerium alloys like $\mathrm{CeCu}_{x} \mathrm{Ni}_{1-x}$ or $\mathrm{CeRh}_{x} \mathrm{Pd}_{1-x}$ by considering the Kondo effect, a ferromagnetic order and a spin glass behavior described by several approaches. The van Hemmen approach gives a good explanation of the properties of cerium alloys and we are describing the magnetic glass clusters which occur in both spin glass and ferromagnetic phases. Third, we present a new description of a frustrated Kondo lattice model, which can account for the behavior under pressure or doping of some ytterbium compounds like $\mathrm{Yb}_{2} \mathrm{Pd}_{2} \mathrm{Sn}$ and $\mathrm{YbAgGe}$.
\end{abstract}

PACS: $71.27 .+\mathrm{a}, 75.10 . \mathrm{Nr}, 75.30 . \mathrm{Nr}$

\section{Introduction to Kondo lattice models}

The physics of strongly correlated electron systems and the Kondo effect were extensively studied in the last fifty years [1]. It is well established that the competition between the Kondo effect and magnetism plays a very important role in anomalous rare-earth and actinide systems [2-6]. In fact, the $4 f$-electrons are well localized and cerium compounds are either Kondo or mixed valence ones. The $5 f$-electrons in actinides are less localized than the $4 f$ electrons of rare-earth systems and it results that there is often an intermediate state between localized and delocalized $5 f$-electrons in many actinide compounds, especially at the beginning of the actinide series for uranium and neptunium compounds.

The superconductivity observed in cerium and uranium compounds, starting from the discovery of the superconductivity of $\mathrm{CeCu}_{2} \mathrm{Si}_{2}$ by Steglich et al. [7] and extended to actinide compounds like $\mathrm{UPd}_{2} \mathrm{Al}_{3}$ [8]. Americium metal [9] and $\mathrm{PuCoGa}_{5}[10]$ is extensively studied at present $[11,12]$, but we will not discuss this problem here.

The Kondo effect has been initially derived for an impurity by a perturbation calculation [13] and has been then computed by exact calculations [14]. At very low temperatures, a complete screening of the spins $S=1 / 2$ of the localized $4 f$ electrons by the spins $s=1 / 2$ of the conduction electrons and a heavy fermion behavior have

\footnotetext{
* e-mail: bernard.cogblin@u-psud.fr
}

been established [15]. But, in a compound containing cerium or another anomalous rare-earth, there is a strong competition between magnetism and the Kondo effect and this competition has been firstly described by the Doniach diagram [16], which gives, with increasing Kondo interaction $J_{\mathrm{K}}$, a magnetic order, like in $\mathrm{CeAl}_{2}$ and then a heavy fermion behavior without magnetism, like in $\mathrm{CeAl}_{3}$. Moreover, the transition from magnetic order to a non-magnetic heavy fermion state has been experimentally observed with increasing pressure in many cerium compounds, such as $\mathrm{CeAl}_{2}$ or $\mathrm{CeRh}_{2} \mathrm{Si}_{2}$ [4]. A similar effect has been observed in $\mathrm{YbCu}_{2} \mathrm{Si}_{2}[17]$ or in other ytterbium compounds, where the Néel temperature increases rapidly with pressure, in opposite to cerium compounds.

To describe the behavior of the Kondo lattice, we have used the following Hamiltonian:

$$
\begin{aligned}
H & =\sum_{\boldsymbol{k} \sigma} \varepsilon_{\boldsymbol{k}} n_{\boldsymbol{k} \sigma}^{\mathrm{c}}+\sum_{i \sigma} E_{0} n_{i \sigma}^{f}+J_{\mathrm{K}} \sum_{i} \boldsymbol{s}_{i} \cdot \boldsymbol{S}_{i} \\
& +J_{\mathrm{H}} \sum_{\langle i j\rangle} \boldsymbol{S}_{i} \cdot \boldsymbol{S}_{j},
\end{aligned}
$$

where $\varepsilon_{\boldsymbol{k}}$ is the energy of the conduction band, $J_{\mathrm{K}}>0$ is the Kondo coupling between the localized spin $\boldsymbol{S}_{i}$ and the spin $s_{i}$ of a conduction electron at the same site and $J_{\mathrm{H}}$ is the interaction between nearest-neighboring localized spins. We choose here $J_{\mathrm{H}}$ to be either positive or negative for antiferromagnetic or ferromagnetic intersite interactions. The Hamiltonian given by Eq. (1) is quite general and we have chosen different forms to treat the different physical cases, as we will explain in each case. 
We have used also the same type of mean field approximation that we will explain later on [18-21]. We have started by describing the normal Kondo lattice model for $S=1 / 2$ spins and we have considered firstly here the case of choosing $J_{\mathrm{H}}$ to be positive, implying that intersite interactions are antiferromagnetic, as it is the case of most cerium compounds [20-22]. We have studied the Kondo effect and the short-range magnetic correlations by considering respectively the two mean field operators $\lambda=\left\langle c_{i \sigma}^{*} f_{i \sigma}\right\rangle$ and $\Gamma=\left\langle f_{i \sigma}^{*} f_{j \sigma}\right\rangle$. We have computed the Kondo temperature $T_{\mathrm{K}}$ and the correlation temperature $T_{\text {cor }}$ at which the short-range magnetic correlations disappear and we have shown that the Kondo effet occurs for a large $J_{\mathrm{K}}$ value and that it is destroyed by an increase of $J_{\mathrm{H}}$.

The Kondo effect tends also to be destroyed by a decrease of the number of conduction electrons, called the "exhaustion" effect [20, 21, 23]. Then, we have described the competition between the Kondo effect and the antiferromagnetic order, within the same type of mean-field approximation and we have obtained a transition versus $J_{\mathrm{K}}$ from an antiferromagnetic ordering to a Kondo phase, which can account roughly for the effect of pressure in the Doniach diagram [22]. The detailed calculations can be found in the original papers [20, 21]. Thus, the Kondo lattice model explains well the behavior of many cerium, ytterbium or other anomalous rare-earth systems. The effect of pressure is often to change a magnetically ordered cerium compound to a non-magnetic compound with a large heavy-fermion character and an opposite effect has been observed in some ytterbium compounds, in good agreement with theory. A similar variation of the magnetism of $\mathrm{Ce}$ or $\mathrm{Yb}$ in alloys has been also observed when there is a change in the concentration of the matrix.

Recently a new and very interesting effect has been also obtained by tuning the dimensionality of the heavy fermion compound $\mathrm{CeIn}_{3}$ [24]. In fact, artificial superlattices of the antiferromagnetic heavy fermion compound $\mathrm{CeIn}_{3}$ and of the conventional non-magnetic metal $\mathrm{LaIn}_{3}$ have been experimentally studied. The heavy fermion compound $\mathrm{CeIn}_{3}$ exhibits an antiferromagnetic ordering at the Néel temperature $T_{\mathrm{N}}=10 \mathrm{~K}$, which is destroyed by applying pressure. By reducing the thickness of the $\mathrm{CeIn}_{3}$ layers, the Néel temperature decreases and finally the magnetic order was suppressed; similarly, the Fermi liquid coefficient of the low temperature resistivity increases. This very interesting result is finally interpreted by saying that the decrease of the dimensionality from $3 \mathrm{D}$ to $2 \mathrm{D}$ has the same effect as the increase of pressure, giving, therefore, a decrease and finally the disappearance of the magnetic ordering [24]. This effect is very fascinating and can be reproduced in other heavy fermion compounds.

In the next sections, we will present recent works using the Kondo lattice models. First, the underscreened Kondo lattice (UKL) model is described to account for the Kondo-magnetism coexistence occurring in some uranium and neptunium compounds [25] with a special em- phasis on a recent improvement able to account for the beginning of the delocalization of the $5 f$-electrons [26]. The following section is devoted to the competition between magnetism, a spin glass state and the Kondo effect [27] and finally the last section gives a brief review on a new calculation on a frustrated Kondo lattice which gives two non-magnetic phases, the Kondo one and a frustrated valence bond solid state [28].

\section{The underscreened Kondo lattice model applied to actinide compounds}

The Kondo lattice (KL) model has been, therefore, recognized to be an appropriate tool for describing the physics of intermetallic strongly correlated electron systems with rare earths or actinides. But, in actinide compounds, the $5 f$-electrons have generally a spin larger than $S=1 / 2$ and moreover they are less localized than the $4 f$-electrons of rare-earths. In fact, it is often difficult to decide, on the basis of the experimental data in actinide systems, between a local Kondo behavior corresponding to a $5 f^{n}$ configuration and a mixed-valence situation. But here, we restrict our study to the case of the Kondo actinide compounds.

Thus, we present here a study of the coexistence between ferromagnetic order and Kondo behavior in actinide compounds. Such a coexistence has been observed in $\mathrm{UTe}$ [29], $\mathrm{UCu}_{0.9} \mathrm{Sb}_{2}$ [30], $\mathrm{UCo}_{0.5} \mathrm{Sb}_{2}$ [31], $\mathrm{UCu}_{2} \mathrm{Si}_{2}$ [32] and also in neptunium compounds $\mathrm{NpNiSi}_{2}$ [33] and $\mathrm{Np}_{2} \mathrm{PdGa}_{3}$ [34]. All these systems undergo a ferromagnetic transition at relatively high Curie temperatures: $T_{\mathrm{C}}=102 \mathrm{~K}(\mathrm{UTe}), T_{\mathrm{C}}=113 \mathrm{~K}\left(\mathrm{UCu}_{0.9} \mathrm{Sb}_{2}\right)$, $T_{\mathrm{C}}=64.5 \mathrm{~K}\left(\mathrm{UCo}_{0.5} \mathrm{Sb}_{2}\right), T_{\mathrm{C}}=103 \mathrm{~K}\left(\mathrm{UCu}_{2} \mathrm{Si}_{2}\right)$, $T_{\mathrm{C}}=51.5 \mathrm{~K}\left(\mathrm{NpNiSi}_{2}\right)$ and $T_{\mathrm{C}}=62.5 \mathrm{~K}\left(\mathrm{~Np}_{2} \mathrm{PdGa}_{3}\right)$. Above the ordering temperature, these materials exhibit a Kondo-like logarithmic decrease of the electrical resistivity, indicating a Kondo behavior. This coexistence and the large Curie temperatures are clearly new features that cannot be explained by the standard KL model, which gives relatively small Curie temperatures in cerium compounds, typically of order $5 \mathrm{~K}$. Uranium monochalcogenides provide a good experimental example showing that the $5 f$ electrons lie between localized and itinerant. US lies closest to the itinerant side for the $5 f$-electrons and USe is in the middle, while UTe is the closest to the localized side and has relatively well localized $5 f$ electrons [35-37]. The ferromagnetic uranium monochalcogenides UTe, USe, and US have been studied at very high pressures. The Curie temperature of UTe increases with pressure up to a maximum at roughly $7 \mathrm{GPa}$ and then decreases with larger pressures $[38,39]$. On the opposite, the Curie temperature of US is continuously decreasing with pressure and $T_{\mathrm{c}}$ of USe remains constant up to $10 \mathrm{GPa}$ and decreases rapidly at higher pressures [38].

Thus, we have proposed a UKL model which consists of a periodic lattice of magnetic atoms with $S=1$ spins, corresponding to the $5 f^{2}$ configuration and interacting with conduction electron spins $s=1 / 2$ via an on-site 
Kondo coupling $J_{\mathrm{K}}[25]$. In addition, the localized spins at neighbouring sites interact ferromagnetically with each other. In this case the Kondo effect does not lead to a complete screening of the localized spins, and the ferromagnetic exchange between the underscreened spins may lead to the formation of a ferromagnetic order. We discuss here, as in Ref. [25], only the case of $S=1$ spins, corresponding to the $5 f^{2}$ configuration. Indeed, it would be necessary to consider also $S=3 / 2$ or $S=2$ spins to discuss different configurations of uranium and neptunium compounds.

The underscreened Kondo model has been well studied for the case of one impurity $[40,41]$. Also, the dual nature of the $5 f$ electrons, assuming two localized $5 f$ electrons and one delocalized one, has been considered by Zwicknagl et al. [42] who have obtained by band calculations a mass enhancement factor in good agreement with experiment in $\mathrm{UPt}_{3}$ and $\mathrm{UPd}_{2} \mathrm{Al}_{3}$ and by Schoenes et al. [29] who have carefully analyzed the variation of the localization of the $5 f$-electrons with concentration and pressure in dilute US and UTe.

We will present now our results on the UKL model. There are two steps in this calculation. The first one is to derive the UKL model with $S=1$ localized spins in a model where the $5 f$ electrons are relatively well localized [25]. The second step is to start from the underscreened Anderson lattice (UAL) model and to use the Schrieffer-Wolff transformation to obtain a finite $f$-band and to describe a possible delocalization of the $5 f$ electrons [26]. All calculations can be found in Refs. [25, 26].

In the UKL model [25], the two $5 f$-electrons are bound into a spin $S_{f}=1$. The starting Hamiltonian is given by Eq. (1), with the following essential difference: the localized spins are here $S=1$ and the second term of the Hamiltonian (1) can be written now as $\sum_{i \sigma \alpha} E_{0} n_{i \sigma}^{f_{\alpha}}$, in order to describe the energy $E_{0}$ of the two $5 f$ localized electrons (defined by the notation $\alpha=1,2$ ) of the $5 f^{2}$ configuration of uranium atoms. Then, the intersite interaction is ferromagnetic with $J_{\mathrm{H}}<0$.

We use here the mean field approximation which has been extensively used for the normal Kondo lattice model and which is appropriate to describe the competition between Kondo effect and magnetic ordering in the Kondo lattice [18-20].

In the UKL model of Ref. [25], we have used the mean field approximation by introducing the Kondo operators

$$
\widehat{\lambda}_{i \sigma}=\sum_{\alpha} \hat{\lambda}_{i \sigma}^{\alpha}=\sum_{\alpha}\left\langle c_{i \sigma}^{*} f_{i \sigma}^{\alpha}\right\rangle
$$

which couples conduction and $f$ electrons at the same site.

Moreover, in order to describe the magnetic properties of the system, we introduce also the magnetization operators for both $f$ - and $c$-electrons

$$
M_{i}=S_{i}^{z}=\frac{1}{2}\left(n_{i \uparrow}^{f}-n_{i \downarrow}^{f}\right),
$$

$$
m_{i}=\sigma_{i}^{z}=\frac{1}{2}\left(n_{i \uparrow}^{\mathrm{c}}-n_{i \downarrow}^{\mathrm{c}}\right) .
$$

The non-zero values of $\langle M\rangle$ and $\langle m\rangle$ describe the magnetic phase, while a non-zero $\lambda_{\sigma}$ describes the Kondo effect and the formation of the heavy-fermion state. The detailed calculations can be found in Ref. [25].

The two temperatures $T_{\mathrm{C}}$ and $T_{\mathrm{K}}$ have been computed within the UKL model [25] and a ferromagnetic-Kondo phase diagram has been obtained as a function of $J_{\mathrm{K}}$. For a finite value of $J_{\mathrm{H}}, T_{\mathrm{C}}$ starts from a finite value at $J_{\mathrm{K}}=0$ and increases continuously versus $J_{\mathrm{K}}$. On the other hand, there is no Kondo effect for small values of $J_{\mathrm{K}}$, and at a critical value $J_{\mathrm{K}}^{\mathrm{c}}$ of $J_{\mathrm{K}}$ (typically of order $0.5-0.6 D$, where $D$ is the half band-width of the conduction band), the Kondo temperature $T_{\mathrm{K}}$ increases very rapidly and crosses the curve of $T_{\mathrm{C}}$ and continues to increase above it. We have, therefore, obtained that ferromagnetism and Kondo effect coexist for a strong Kondo coupling value and we have obtained a "ferromagnetic-Kondo phase diagram" with regions of pure ferromagnetism and of Kondo-ferromagnetism coexistence when the parameter $J_{\mathrm{K}}$ increases.

The previous calculations assume that the $5 f$ electrons are relatively well localized and that the actinide ions are well described by $S=1$ localized spins. But, as already described above, the $5 f$ electrons are often in-between localized and itinerant. In order to describe Kondo effect in such weakly delocalized $5 f$ electrons, we have considered the UAL model for the case of a twice degenerate $f$ orbital with two $5 f$ electrons coupled with an itinerant band [26]. Because of intra-atomic Hund's coupling, the two $5 f$ electrons are coupled in the triplet state $S=1$ in their ground state. We take, as usual, the $f-c$ hybridization term for the two $f$-levels and we derive the Schrieffer-Wolff transformation which is clearly new for the present case of two $f$-states [26]. Detailed calculations can be found in Ref. [26]. The main result is that we obtain now a finite and narrow $5 f$ bandwidth, in addition to the exchange Kondo-like usual interaction.

The half $f$-bandwidth derived by the Schrieffer-Wolff transformation is spin-dependent and it is given by

$$
A_{\sigma}=-\frac{J_{\mathrm{K}} P}{16}\left[7+3\left(M^{f}\right)^{2}+6 \sigma M^{f}\right],
$$

where $\sigma$ is equal here to \pm 1 . We see also that the effective bandwidth and the $f$-magnetization $M_{f}$ are correlated.

Finally, we study now the parameter dependence of the effective bandwidth $W_{f}$ and therefore we consider the following definitions of $W_{f}$ :

— case (a): a constant bandwidth: $W_{f}=$ const;

- case (b): a bandwidth $W_{f}$ proportional to the Kondo coupling constant: $W_{f}=Q J_{\mathrm{K}}$; in this way we can take into account the effect of pressure on both the bandwidth and the Kondo coupling, since both are functions of the increase of hybridization under pressure. 
- case (c): a spin-dependent bandwidth directly obtained from the SW transformation $W_{f}=A_{\sigma}$, according to the previous discussion. $P$ is an adjustable parameter.

We have plotted in Fig. 1 the two temperatures $T_{\mathrm{C}}$ and $T_{\mathrm{K}}$ versus $J_{\mathrm{K}}$ for the three previously defined cases. We obtain a very rapid increase of $T_{\mathrm{K}}$ above a critical value $J_{\mathrm{K}}^{\mathrm{c}}$ of $J_{\mathrm{K}}$, while $T_{\mathrm{C}}$ has a smoother variation. Concerning the Kondo effect, a peculiar behavior has been obtained for values of $J_{\mathrm{K}}$ just above the crossing point: at the temperature $T_{1}$, the Kondo effect vanishes and is non zero-only between $T_{1}$ and $T_{\mathrm{K}}$, as explained in Ref. [26].

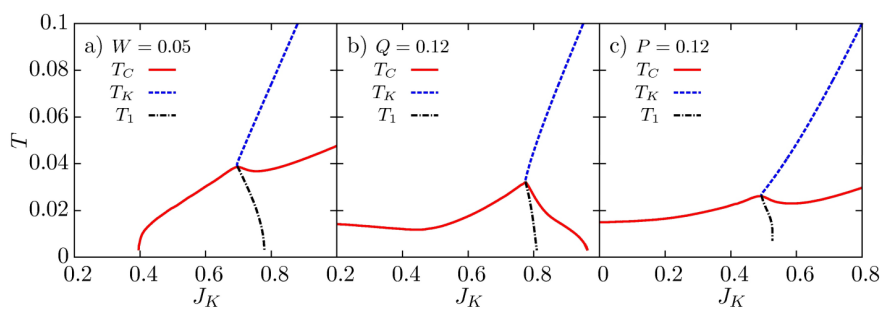

Fig. 1. Curie temperature $T_{\mathrm{C}}$ and Kondo temperature $T_{\mathrm{K}}$ versus $J_{\mathrm{K}}$ for the three cases (a), (b) and (c), with $J_{\mathrm{H}}=-0.01, n^{\mathrm{c}}=0.8$ and $n_{\text {tot }}^{f}=2[26]$.

But the most important result is that $T_{\mathrm{C}}$ is passing through a maximum with increasing $J_{\mathrm{K}}$, as shown in particular for the case (b). Since $J_{\mathrm{K}}$ increases with pressure, the present model can account very well for the observed pressure dependence of the Curie temperature in UTe compound. So, we have shown that our model includes two effects which are essential to describe the $5 f$-electron compounds: the small delocalization of the $5 f$-electrons, and the $S=1$ spins found in uranium or neptunium compounds. The first effect works against magnetism, while the second one favors magnetism. The competition between these two effects leads to complex phase diagrams which can improve the description of some actinide compounds and explain in particular the maximum of $T_{\mathrm{C}}$ observed experimentally in UTe compound with increasing pressure.

We have also studied the opposite case where $T_{\mathrm{C}}$ is larger than $T_{\mathrm{K}}$ and we have obtained a rapid decrease of the magnetization at a low temperature corresponding roughly to $T_{\mathrm{K}}$ well below the Curie temperature. This effect has been observed in the experimental magnetization curve of neptunium compound $\mathrm{NpNiSi}_{2}$ [33] and our model explains well the experimental behavior.

The resulting "ferromagnetic-Kondo" diagram, which is given in Refs. [25, 26], is completely different from the Doniach diagram derived for the Kondo lattice model appropriate for cerium or ytterbium compounds and represents really a new result for the study of magnetic actinide compounds.

The initial increase of $T_{\mathrm{C}}$ with pressure is clearly explained by our first model [25], since $J_{\mathrm{K}}$ increases with increasing pressure. On the other hand, the maximum and the decrease of $T_{\mathrm{C}}$ at higher pressure is explained by the UKL model with a weak delocalization of the $5 f$ electrons [26]. Similarly, the decrease of $T_{\mathrm{C}}$ in US compound corresponds to a continuous delocalisation of the $5 f$ electrons which are already itinerant at normal pressure. The case of USe is intermediate, with $5 f$ electrons between localized and itinerant at normal pressure. Sheng and Cooper [35] have performed $a b$ initio band calculations which can account for the pressure dependence of $T_{\mathrm{C}}$ in uranium monochalcogenides.

We can presently conclude that the behavior of $T_{\mathrm{C}}$ and $T_{\mathrm{K}}$ shown in Fig. 1 [26] presents two important differences with respect to the previous plots obtained with the UKL model [25]: first, $T_{\mathrm{C}}$ can pass through a maximum above roughly $J_{\mathrm{K}}^{\mathrm{c}}$ and decreases after with increasing $J_{\mathrm{K}}$; second, in a certain $J_{\mathrm{K}}$ range, two values of $T_{\mathrm{K}}$ are obtained and $\lambda$ is non-zero only between these two temperatures. The first effect can account for the maximum of $T_{\mathrm{C}}$ observed in UTe at high pressures. The second effect is connected to the fact that the Kondo screening of $S=1$ spins cannot be completed at very low temperatures [40] and such an effect has been observed, for the first time, in the experimental magnetization curve of neptunium compound $\mathrm{NpNiSi}_{2}$ [33], which presents a rapid drop at low temperatures. Indeed, the influence of the Kondo effect has to be studied in detail there and anyway these results will be discussed elsewhere soon [43].

\section{The spin glass-Kondo-magnetic order competition}

There are many compounds with cerium, ytterbium, uranium, other anomalous rare-earths or actinides, which have a Kondo behavior with the classical Fermi liquid behavior at very low temperatures and eventually a non-Fermi liquid one. But in fact, there are not so many compounds which present together a Kondo phase, a spin glass one and a magnetic (ferromagnetic or antiferromagnetic) ordering. Such a competition has been observed in disordered cerium or uranium alloys and here we will discuss some examples of these systems. First, $\mathrm{CeNi}_{x} \mathrm{Cu}_{1-x}$ alloys have been extensively studied and the transition from spin glass to ferromagnetism occurs with decreasing temperature at a given concentration and recent works on these alloys show the existence of a new Kondo cluster spin glass state [44-48]. Another example is provided by the $\mathrm{CeRh}_{x} \mathrm{Pd}_{1-x}$ alloys [49, 50], where a careful experimental study shows the presence of the Kondo clusters near $x=0.8$ close to a quantum critical point (QCP) when the ferromagnetic phase is very close to disappear [49].

We will present here some features on the spin glass-Kondo-magnetic order and we describe firstly the case of the well known $\mathrm{CeNi}_{x} \mathrm{Cu}_{1-x}$ alloys, that we have extensively studied, and finally we discuss briefly the other cases.

The $\mathrm{CeNi}_{x} \mathrm{Cu}_{1-x}$ alloys present, at low temperatures, an antiferromagnetic order for low $\mathrm{Ni}$ concentration 
( $x$ small) and a Kondo behavior for low $\mathrm{Cu}$ concentration ( $x$ large). But, in the intermediate concentration range, typically for $x$ between 0.4 and 0.7 , it was firstly shown that, when temperature decreases, there are successively a spin-glass phase and then a ferromagnetic one $[44,45]$. Very recently, these alloys have been investigated by $\mu \mathrm{SR}$ spectroscopy, small angle neutron scattering (SANS) and magnetic experiments leading to the determination of hysteresis loops [46-48]. The proposed phase diagram in the intermediate concentration range consists firstly in the presence of an intermediate magnetic state where dynamic clusters develop with decreasing temperature below the paramagnetic state; then, the volume fraction of these clusters increases as temperature decreases and they become frozen at very low temperatures. Thus, recent experiments have shown the occurrence of an inhomogeneous "cluster glass" (CG), followed, with decreasing temperature, by an inhomogeneous ferromagnetic (IFM) order, with a percolative evolution of the clusters from the CG state to the IFM state [47, 48].

On the other hand, there has been an extensive theoretical work to understand the complicate competition between the Kondo effect, the spin glass (SG) and magnetic orderings in such disordered cerium alloys. The calculation starts from a Hamiltonian similar to that given by Eq. (1), including the same intra-site Kondo interaction term with an exchange integral $J_{\mathrm{K}}$ and a different last term corresponding to the spin glass phase given by

$$
H_{\mathrm{SG}}=\sum_{i, j} J_{i j} S_{f i}^{z} S_{f j}^{z} .
$$

We have performed different calculations for the spin glass-Kondo interaction and the main difference between them lies in the approach used to describe the spin glass.

In our first calculation, we have taken the SherringtonKirkpatrick model [51] for the exchange intersite integrals which are assumed to be randomly distributed with a Gaussian distribution. The first model assumes a zero mean value of the Gaussian distribution in order to study only the Kondo-spin glass interaction and we have, therefore, obtained a phase diagram showing first a spin glass phase and then a Kondo phase with increasing the Kondo exchange parameter $J_{\mathrm{K}}$ [52]. Then, in order to obtain a more complex phase diagram with a ferromagnetic [53] or an antiferromagnetic [54] phase occurring at low temperatures for small $J_{\mathrm{K}}$ values, we have taken the same model but with a non-zero mean value $J_{0}$ of the Gaussian distribution. Thus, it results that the Kondo phase obtained for large $J_{\mathrm{K}}$ values is still there, but that there is a competition between the spin glass phase and the magnetic phase for smaller $J_{\mathrm{K}}$ values. However, in the ferromagnetic case, when $J_{\mathrm{K}}$ is typically of order $J_{0}$, we have obtained, with decreasing temperature, successively a ferromagnetic phase, then a mixed ferromagnetic-spin glass phase and finally a spin glass phase. The evidence of a spin glass phase at very low temperatures is in disagreement with experimental results previously described for $\mathrm{CeNi}_{x} \mathrm{Cu}_{1-x}$ alloys.
Thus, we have then considered another spin glass approach which introduces a generalization of the Mattis model [55] and which represents an interpolation between ferromagnetism and a highly disordered spin glass [56]. For a reasonable value of the relative importance of the ferromagnetic and spin glass phases, we have obtained, with decreasing temperature, a spin glass phase, then a mixed ferromagnetic-spin glass phase and finally a ferromagnetic phase with decreasing temperature for relatively small $J_{\mathrm{K}}$ values. The Kondo phase is always present for large $J_{\mathrm{K}}$ values. This theoretical result accounts better for the experimental phase diagram of $\mathrm{CeNi}_{x} \mathrm{Cu}_{1-x}$ alloys, with in particular at low temperatures the ferromagnetic phase below the spin glass phase.

Finally, we have used recently the van Hemmen model [57] to describe the interplay of the Kondo effect, spin glass and ferromagnetism in disordered Ce alloys. This model gives a good description of this interplay and appears to be simpler than the previous models, in particular for the treatment of clusters, as we will see later on [27].

Thus, in order to improve again the agreement with the experimental case of $\mathrm{CeNi}_{1-x} \mathrm{Cu}_{x}$ alloys, we introduce here a new kind of disordered coupling $J_{i j}$ given by van Hemmen (vH) [57] as

$$
J_{i j}=\frac{J}{N}\left(\xi_{i} \eta_{j}+\eta_{i} \xi_{j}\right)+\frac{I_{0}}{N},
$$

where $\xi_{i}$ and $\eta_{j}$ are equal to \pm 1 and are random variables which follow a bimodal distribution [27]. We take here both a random SG contribution and a ferromagnetic (FM) one respectively proportional to the parameters $J$ and $I_{0}$. Our calculations using the $\mathrm{vH}$ approach have given different phase diagrams which depend on the ratio $I_{0} / J$. Several cases are described in Ref. [27] and we have obtained the typical case of a transition with decreasing temperature from spin glass (SG) to ferromagnetism (FM) with an intermediate "mixed" phase $\mathrm{SG}+\mathrm{FM}$ for small $J_{\mathrm{K}}$ values and still the Kondo phase for large $J_{K}$ values [27]. The use of the van Hemmen model offers some advantages: first the calculation does not need the use of the replica method and is simpler; second there exists a real mixed phase between spin glass and ferromagnetism, which is in good agreement with the experimental phase diagram of $\mathrm{CeNi}_{x} \mathrm{Cu}_{1-x}$ alloys [27]. Thus, the more "local" description [27] given by the van Hemmen model seems to be more adequate here than the "average" description used for example in the Sherrington-Kirkpatrick approach [51, 53].

However, a real improvement will be provided by trying to describe better the clusters which are yielding the cluster-glass state (CG) with ferromagnetic correlations and the IFM state and also the observed percolative CG-IFM transition observed with decreasing temperature $[46,47]$. A first approach had been proposed to obtain clusters with intrasite Kondo interactions and both intra- and inter-cluster magnetic interactions within the Sherrington-Kirkpatrick approach [58].

More recently, a Kondo cluster-glass model for spin 
glass cerium alloys, like $\mathrm{CeNi}_{x} \mathrm{Cu}_{1-x}$ or $\mathrm{CeRh}_{x} \mathrm{Pd}_{1-x}$, has been proposed using the $\mathrm{vH}$ model [59]. This model starts from the Kondo and ferromagnetic intra-cluster interactions and an inter-cluster $\mathrm{vH}$ interaction given by Eq. (6). The intra-cluster interactions are treated exactly by diagonalisation and the inter-cluster interaction is treated by the mean field approximation, as previously used for the $\mathrm{vH}$ model. The number of atoms $n_{\mathrm{s}}$ inside each cluster is presently limited to 4 or 5 [59]. However, for relatively large values of $J_{\mathrm{K}}$ and $I_{0}$, we have obtained successively with decreasing temperature a Kondo phase followed by a Kondo cluster spin glass where both the Kondo and the cluster spin glass (CG) order parameters are non-zero; finally a mixed Kondo-CG-ferromagnetic phase can be obtained at very low temperatures. Thus, the transition with decreasing temperature is rather smooth and can be interpreted as a percolative one, which can present an account for the experimental percolative CG-IFM transition observed in disordered cerium alloys [48, 59].
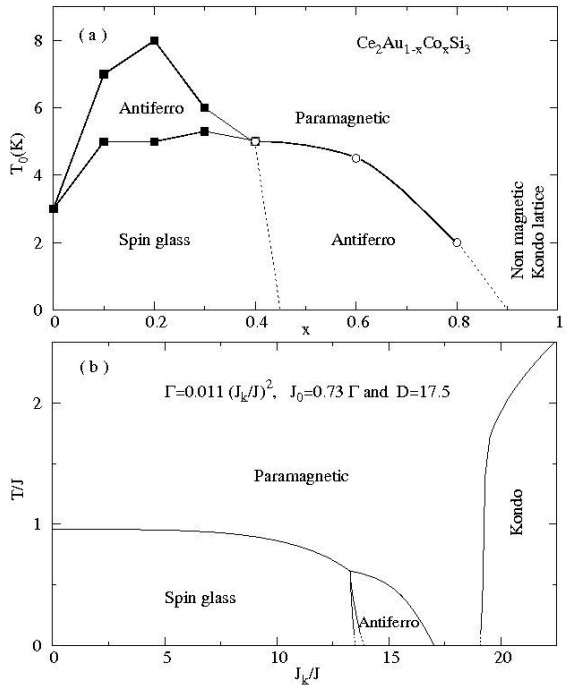

Fig. 2. Comparison between experimental and theoretical phase diagrams: the upper figure is the experimental phase diagram of $\mathrm{Ce}_{2} \mathrm{Au}_{1-x} \mathrm{Co}_{x} \mathrm{Si}_{3}$ alloys [60]; the lower figure is a theoretical phase diagram $T / J$ versus $J_{\mathrm{K}} / J$ for the relations $\Gamma=0.011\left(J_{\mathrm{K}} / J\right)^{2}$ and $J_{0} / J=0.73 \Gamma / J$, where the dotted lines are the extrapolations carried to lower temperatures [61].

After the description of the well studied case of the $\mathrm{CeNi}_{x} \mathrm{Cu}_{1-x}$ alloys, we will discuss the behavior of a spin glass-Kondo-magnetic order competition in $\mathrm{Ce}_{2} \mathrm{Au}_{1-x} \mathrm{Co}_{x} \mathrm{Si}_{3}$ alloys; these alloys present versus the concentration $x$ a transition from spin glass to an antiferromagnetic order and finally to a Kondo phase, as shown in the experimental (upper) part of Fig. 2 [60]. We have studied theoretically the behavior of such a system by taking two Kondo sublattices with an intrasite Kondo interaction given by $J_{\mathrm{K}}$, an interlattice quantum Ising interaction and a transverse field $\Gamma[61]$. The interlattice coupling is a random Gaussian distributed variable, with an average and a variance given respectively by $2 J_{0}$ and $32 J^{2}$. We have taken here the assumption of choosing both $J_{0} / J$ and $\Gamma / J$ proportional to $\left(J_{\mathrm{K}} / J\right)^{2}$; this assumption which has been already used in Ref. [20] allows to describe the variation of the intersite interaction or the Néel temperature as coming from the Ruderman-KittelKasuya-Yosida (RKKY) interaction. One consequence of this choice is to obtain an increase of the intersite interaction with increasing $J_{\mathrm{K}}$ and consequently to favour magnetism and reduce the increase of the Kondo effect when $J_{\mathrm{K}}$ increases.

Figure 2 gives a comparison between the experimental phase diagram of $\mathrm{Ce}_{2} \mathrm{Au}_{1-x} \mathrm{Co}_{x} \mathrm{Si}_{3}$ alloys and the theoretical phase diagram giving $T / J$ versus $J_{\mathrm{K}} / J$ obtained with the previous calculation. We have taken here reasonable parameters $\Gamma=0.011\left(J_{\mathrm{K}} / J\right)^{2}$ and $J_{0}=$ $0.73 \Gamma$ [61]. The agreement between the theoretical curves and the experimental phase diagram of $\mathrm{Ce}_{2} \mathrm{Au}_{1-x} \mathrm{Co}_{x} \mathrm{Si}_{3}$ alloys is relatively good and gives an improvement of this model with respect to previous ones. However, the relationship between the parameters of the model and the matrix concentration is certainly less clear than the pressure dependence and the assumed dependence of the intersite interaction in $\left(J_{\mathrm{K}}\right)^{2}$ can also be subject to discussion.

Another cases are provided by disordered uranium alloys such as $\mathrm{UCu}_{5-x} \mathrm{Pd}_{x}$ [62], $\mathrm{U}_{1-x} \mathrm{Y}_{x} \mathrm{Pd}_{2} \mathrm{Al}_{3}$ [63] or $\mathrm{U}_{1-x} \mathrm{La}_{x} \mathrm{Pd}_{2} \mathrm{Al}_{3}$ [64]. When the concentration $x$ increases, one observes first an antiferromagnetic order, then a spin glass state and finally a non-Fermi liquid Kondo behavior. A non-Fermi liquid behavior has been also observed in the Kondo alloys $\mathrm{Y}_{1-x} \mathrm{U}_{x} \mathrm{Pd}_{3}$ [65, 66]. Other interesting cases, like $\mathrm{Ce}_{2} \mathrm{NiGe}_{3}$ [67], $\mathrm{U}_{3} \mathrm{Co}_{4+x} \mathrm{Al}_{12-x}$ [68], $\mathrm{U}_{3} \mathrm{Rh}_{3} \mathrm{Sb}_{4}$ [69] or $\mathrm{UCu}_{2} \mathrm{Si}_{2-x} \mathrm{Ge}_{x}$ [70] can have also a competition between a Kondo behavior, a spin glass one and a magnetic ordering.

Indeed, there was a very important experimental and theoretical research in the field of spin glasses [71], but the presence of the Kondo effect and the magnetic order in spin glass disordered alloys occurs mainly in cerium or uranium alloys, as we have seen in this section. However, several questions are still under discussion. First, the problem of clusters and in particular the increase of the size of the clusters with decreasing temperature are still under present study. Second, the influence of an applied random field on the spin glass-Kondo-magnetic disordered alloys is presently theoretically studied [58], in order to improve the description of recent experiments performed in $\mathrm{CeNi}_{x} \mathrm{Cu}_{1-x}$ alloys [72, 73]. Third, the difference of behavior between cerium or uranium alloys or consequently between the different Kondo models is not clearly established at present, but it is important to consider this point. Fourth, the position of the spin glass phase in the phase diagram with respect to the other phases is also a very interesting question [74]. Finally, the role of the spin glass in the Kondo lattice has been well studied and continues to be very attractive. 


\section{Frustration in the Kondo lattice model}

The frustration in many systems, especially in magnetic ones, has been extensively studied [75] and here a recent work on frustrated magnetic Kondo compounds will be presented briefly with an extension of the Doniach phase diagram to include frustration effects [28]. A qualitative phase diagram had been already presented [76], but here we present a more quantitative derivation of a frustrated Kondo lattice which can describe the behavior of heavy fermion compounds and in particular of ytterbium compounds as a function of pressure. Detailed calculations can be found in the paper of Bernhard et al. [28].

The Kondo lattice problem is studied here in the Shastry-Sutherland geometry [77]. The starting Hamiltonian contains several terms as in Eq. (1): a conduction band term, a Kondo term on each site and a Heisenberg lattice term between localized spins $S_{i}$ on different sites with exchange interactions $J_{i j}$. In the ShastrySutherland lattice [77] before introducing the Kondo effect, the most important point is that one takes two different positive values $J$ and $J^{\prime}$ when sites $i$ and $j$ are connected respectively by a diagonal or a non-diagonal bond in a lattice equivalent to a square lattice. But in fact, in some compounds, the lattice is distorted, the distance along the diagonal is reduced and the corresponding $J$ value becomes larger than $J^{\prime}$. For strong frustration, $J \gg J^{\prime}$, the ground state consists of singlet dimers formed along the diagonal bonds, while, for weak frustration, $J \ll J^{\prime}$, the ground state is a two-sublattice antiferromagnetic state on the square lattice.

Here, we introduce now the Kondo effect on each site and, in the mean field approximation, we take 3 solutions: the antiferromagnetic (AF) solution with finite magnetizations for both localized and conduction electrons, the valence bond solid (VBS) phase with non-zero values of $\left\langle f_{j \sigma}^{*} f_{i \sigma}\right\rangle$, where $i$ and $j$ are along the diagonal bond and finally the Kondo $(\mathrm{K})$ phase with essentially, as usual, a non-zero value of $\left\langle f_{i \sigma}^{*} c_{i \sigma}\right\rangle$, but also small non-zero values of $\left\langle f_{j \sigma}^{*} f_{i \sigma}\right\rangle$ with $i$ and $j$ along the diagonal or non-diagonal bonds. The conduction band is described in the tight-binding approximation by hopping integrals $t_{i j}$, which are defined by the two values $t$ and $t^{\prime}$ along diagonal or non-diagonal bonds. The VBS phase is a frustrated one without magnetic order and Kondo effect and represents a new phase in the magnetic phase diagram [28]. Only these three phases have been taken here and we do not study the possibility of a superconducting phase [76] or eventually of a mixed AF-K phase.

The zero temperature phase diagram of the ShastrySutherland Kondo lattice model has been determined and is presented in Fig. 3 as a function of the Kondo parameter $J_{\mathrm{K}} / t^{\prime}$ and the frustration parameter $J / J^{\prime}$ for a number of conduction electrons $n_{\mathrm{c}}=0.9$ [28]. We see that there is a direct transition from the VBS phase to the Kondo one versus $J_{\mathrm{K}} / t^{\prime}$ for a large frustration parameter $J / J^{\prime}$ and this transition is a first-order one. On the opposite, for a smaller frustration parameter $J / J^{\prime}$, there

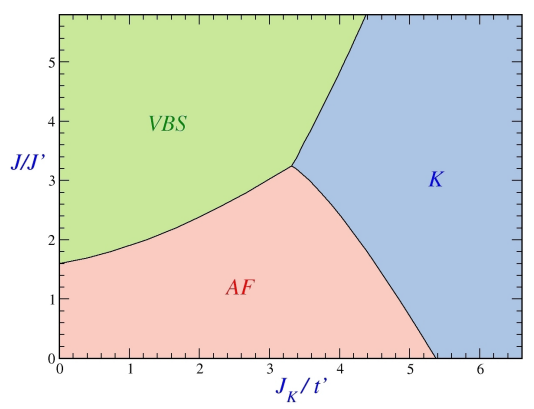

Fig. 3. Phase diagram of the Shastry-Sutherland Kondo lattice model for $J^{\prime}=0.5 t^{\prime}, t=0.1 t^{\prime}$ and $n_{\mathrm{c}}=0.9$, as explained in the text [28].

are two transitions from VBS to AF and from AF to Kondo versus $J_{\mathrm{K}} / t^{\prime}$. For $J_{\mathrm{K}}=0$, the transition between $\mathrm{AF}$ and VBS phases occurs at $J / J^{\prime}=1.6$, which is very close to the value previously obtained for the $S=1 / 2$ Shastry-Sutherland model without any Kondo effect.

The two phases VBS and $\mathrm{K}$ shown on the phase diagram of Fig. 3 are both non-magnetic, but they are deeply different because the VBS phase corresponds to the coupling of localized spins on 2 sites along the diagonal to give singlet dimers, while magnetism disappears in the Kondo phase as due to the local screening of the localized spins on each site by the conduction electrons; in particular, the VBS phase does not have the properties of the Kondo phase and must have a small electronic specific heat constant. For large enough $J_{K}$, we recover, as usual, a Kondo phase.

Thus, the consideration of the frustration in the Shastry-Sutherland Kondo lattice model gives a new non-magnetic phase for small $J_{\mathrm{K}}$ values. It is well known that the increase of pressure increases $J_{\mathrm{K}} / t^{\prime}$ in cerium compounds and decreases it in ytterbium compounds. The phase diagram shown in Fig. 3 can, therefore, account for the pressure dependence of some ytterbium compounds [28] and we present two clear examples provided by $\mathrm{Yb}_{2} \mathrm{Pd}_{2} \mathrm{Sn}[78]$ and $\mathrm{YbAgGe}[79]$.

The compound $\mathrm{Yb}_{2} \mathrm{Pd}_{2} \mathrm{Sn}$, which has the topology of the Shastry-Sutherland lattice, is a non-magnetic Kondo compound with an electronic specific heat constant $\gamma=$ $560 \mathrm{~mJ} /\left(\mathrm{mol} \mathrm{K}^{2}\right)$ at normal pressure and low temperatures. It becomes AF above $1 \mathrm{GPa}$; its Néel temperature increases up to maximum of $1.2 \mathrm{~K}$ at $2.5 \mathrm{GPa}$ and decreases till $4 \mathrm{GPa}$; the compound $\mathrm{Yb}_{2} \mathrm{Pd}_{2} \mathrm{Sn}$ becomes non-magnetic above $4 \mathrm{GPa}$ and we can think that this high pressure non-magnetic phase is a VBS one, according to the present predictions of Fig. 3. A similar result has been obtained for alloys $\mathrm{Yb}_{2} \mathrm{Pd}_{2} \mathrm{In}_{1-x} \mathrm{Sn}_{x}$, where there are successively, with increasing $x$, a non-magnetic phase, then an AF one for $x$ roughly between 0.4 and 0.9 and finally a second non-magnetic phase [78]. Further experimental work is necessary to check the VBS nature of the second non-magnetic phase which is clearly very different from the Kondo phase.

The compound YbAgGe has a different crystallographic structure similar to the kagome geometry, but 
it is strongly frustrated. It is weakly magnetic at normal pressure and the Néel temperature $T_{\mathrm{N}}$ increases up to a maximum of $5.4 \mathrm{~K}$ at $6.8 \mathrm{GPa}$ and then decreases to $2.2 \mathrm{~K}$ at $13.8 \mathrm{GPa}$. The extrapolated zero value of $T_{\mathrm{N}}$ corresponds roughly to a pressure of $16 \mathrm{GPa}$, above which there is again a second non-magnetic phase.

There are presently many other strongly frustrated cerium and ytterbium compounds, like CePdAl [75], $\mathrm{Ce}_{2} \mathrm{Pd}_{2} \mathrm{Sn}[78]$ or $\mathrm{Yb}_{2} \mathrm{Pt}_{2} \mathrm{~Pb}$ [80]. The present study of the frustration in heavy fermion compounds is a positive feature of the Kondo lattice, because it yields the existence of a second non-magnetic phase which originates from the peculiar situation of the coupling of localized spins on 2 sites along the diagonal bond. However, further experimental works are certainly necessary to better identify the nature of this VBS phase, which is clearly different from the non-magnetic Kondo lattice phase.

\section{Conclusions}

Thus, we have presented here Kondo lattice models which can explain different interplays between the Kondo effect and magnetism in many cerium compounds, starting from the coexistence between ferromagnetism and the underscreened Kondo lattice model observed in some uranium and neptunium compounds, then the influence of spin glass on the Kondo lattice in disordered cerium or uranium alloys and finally the influence of frustration on heavy fermion compounds and in particular ytterbium compounds.

In conclusion, there is a lot of works in the field of the interplay between the Kondo effect and magnetism, particularly the different properties of actinide systems which can present a multichannel Kondo effect and the interaction between the spin glass and Kondo states in disordered cerium or uranium alloys or the influence of frustration. We have not discussed here the occurrence of superconductivity in cerium or actinide compounds which is also a fascinating subject. We can also mention the recent and very interesting work of tuning the dimensionality of a heavy fermion compound by reducing the thickness of the layers [24]. Indeed the study of the extremely thin layers or more generally of nanoscale Kondo systems is also extremely promising for the study of heavy fermion physics.

The problem of the hidden order in the compound $\mathrm{URu}_{2} \mathrm{Si}_{2}$ is extensively studied and a phase transition arising from the underscreened Anderson lattice model has been recently presented to explain such a transition in $\mathrm{URu}_{2} \mathrm{Si}_{2}$ [81].

\section{Acknowledgments}

I would like to thank very much my colleagues, B.H. Bernhard, J.C. Gomez Sal, J.R. Iglesias, C. Lacroix, S.G. Magalhaes, N.B. Perkins, A.S. da Rosa Simoes, C. Thomas, R. Troc and F.M. Zimmer for our very good collaborations and very fruitful discussions.

\section{References}

[1] A.C. Hewson, The Kondo Problem to Heavy Fermions, Cambridge Univerity Press, Cambridge 1993.
[2] J.M. Lawrence, P.S. Risenborough, R.D. Parks, Rep. Prog. Phys. 44, 1 (1981).

[3] G.R. Stewart, Rev. Mod. Phys. 56, 755 (1984).

[4] B. Coqblin, AIP Conf. Proc. 846, 3 (2006) and references therein.

[5] J. Custers, P. Gegenwart, H. Wilhelm, K. Neumaler, Y. Tokiwa, O. Trovarelli, C. Geibel, F. Steglich, C. Pepin, P. Coleman, Nature 424, 524 (2003).

[6] Qimiao Si, F. Steglich, Science 329, 1161 (2010).

[7] F. Steglich, J. Aarts, C.D. Bredl, W. Lieke, D. Meschede, W. Franz, H. Schafer, Phys. Rev. Lett. 43, 1892 (1979).

[8] C. Geibel, C. Schank, S. Thies, H. Kitazawa, C.D. Bredl, A. Bohm, M. Rau, A. Grauel, R. Caspary, R. Helfrich, U. Ahlheim, G. Weber, F. Steglich, Z. Phys. B 84, 1 (1991).

[9] J.L. Smith, R.G. Haire, Science 200, 535 (2005).

[10] J.L. Sarrao, L.A. Morales, J.D. Thomson, B.L. Scott, G.R. Stewart, F. Wastin, J. Rebizant, P. Boulet, E. Colineau, G.H. Lander, Nature 420, 297 (2002).

[11] F. Steglich, Physica B 359-361, 326 (2005).

[12] J. Flouquet, Prog. Low Temp. Phys. 15, 139 (2006).

[13] J. Kondo, Prog. Theoret. Phys. (Kyoto) 32, 37 (1964).

[14] K.G. Wilson, Rev. Mod. Phys. 47, 773 (1975).

[15] K. Andres, J.E. Graebner, H. Ott, Phys. Rev. Lett. 35, 1779 (1975).

[16] S. Doniach, in: Proc. Valence Instabilities and Related Narrow-Band Phenomena, Ed. R.D. Parks, Plenum Press, New York 1976, p. 168.

[17] K. Alami-Yadri, H. Wilhelm, D. Jaccard, Physica B 259-261, 157 (1999).

[18] C. Lacroix, M. Cyrot, Phys. Rev. B 20, 1969 (1979).

[19] P. Coleman, N. Andrei, J. Phys., Condens. Matter 1, 4057 (1989).

[20] J.R. Iglesias, C. Lacroix, B. Coqblin, Phys. Rev. B 56, 11820 (1997).

[21] B. Coqblin, C. Lacroix, M.A. Gusmão, J.R. Iglesias, Phys. Rev. B 67, 064417 (2003).

[22] B. Coqblin, M.A. Gusmão, J.R. Iglesias, A.R. Ruppenthal, C. Lacroix, J. Magn. Magn. Mater. 226-230, 115 (2001).

[23] P. Nozières, Eur. Phys. J. B 6, 447 (1998).

[24] H. Shishido, T. Shibauchi, K. Yasu, T. Kato, H. Kontani, T. Terashima, Y. Matsuda, Science 327, 980 (2010).

[25] N.B. Perkins, M.D. Nunez-Regueiro, J.R. Iglesias, B. Coqblin, Phys. Rev. B 76, 125101 (2007).

[26] C. Thomas, A.S. da Rosa Simões, J.R. Iglesias, C. Lacroix, N.B. Perkins, B. Coqblin, Phys. Rev. B 83, 014415 (2011).

[27] S.G Magalhaes, F.M. Zimmer, B. Coqblin, Phys. Rev. B 81, 094424 (2010).

[28] B.H. Bernhard, B. Coqblin, C. Lacroix, Phys. Rev. B 83, 214427 (2011).

[29] J. Schoenes, O. Vogt, J. Lohle, F. Hulliger, K. Mattenberger, Phys. Rev. B 53, 14987 (1996).

[30] Z. Bukowski, R. Troc, J. Stepien-Damm, C. Sulkowski, V.H. Tran, J. Alloys Comp. 403, 65 (2005). 
[31] V.H. Tran, R. Troc, Z. Bukowski, D. Badurski, C. Sulkowski, Phys. Rev. B 71, 094428 (2005).

[32] R. Troc, M. Samsel-Czekala, B. Coqblin, presented at PM'11 Conf. Poznań 2011 and to be published.

[33] E. Colineau, F. Wastin, J.P. Sanchez, J. Rebizant, J. Phys., Condens. Matter 20, 075207 (2008).

[34] V.H. Tran, J.C. Griveau, R. Eloirdi, W. Miiller, E. Colineau, Phys. Rev. B 82, 094407 (2010).

[35] Q.G. Sheng, B.R. Cooper, J. Magn. Magn. Mater. 164, 335 (1996).

[36] J. Schoenes, B. Frick, O. Vogt, Phys. Rev. B 30,6578 (1984).

[37] J. Schoenes, J. Less-Common Met. 30, 6578 (1986).

[38] A.L. Cornelius, J.S. Schilling, O. Vogt, K. Mattenberger, U. Benedict, J. Magn. Magn. Mater. 161, 169 (1996).

[39] P. Link, U. Benedict, J. Wittig, H. Wuhl, J. Phys., Condens. Matter 4, 5585 (1992).

[40] P. Nozières, A. Blandin, J. Phys. (France) 41, 193 (1980).

[41] P. Schlottmann, P.D. Sacramento, Adv. Phys. 42, 641 (1993).

[42] G. Zwicknagl, A.N. Yaresko, P. Fulde, Phys. Rev. B 68, 052508 (2003).

[43] C. Thomas, A.S. da Rosa Simões, J.R. Iglesias, C. Lacroix, B. Coqblin, presented at the SCES2011 Conf. Cambridge (England), 2011, to be published in J. Phys., Conf. Series.

[44] J.C. Gomez Sal, J. Garcia Soldevilla, J.A. Blanco, J.I. Espeso, J. Rodriguez Fernandez, F. Luis, F Bartolomé, J. Bartolomé, Phys. Rev. B 56, 11741 (1997).

[45] J. Garcia Soldevilla, J.C. Gomez Sal, J.A. Blanco, J.I. Espeso, J. Rodriguez Fernandez, Phys. Rev. B 61, 6821 (2000).

[46] N. Marcano, J.I. Espeso, J.C. Gomez Sal, J. Rodriguez Fernandez, J. Herrero-Albillos, F. Bartolome, Phys. Rev. B 71, 134401 (2005).

[47] N. Marcano, J.C. Gomez Sal, J.I. Espeso, J.M. De Teresa, P.A. Algarabel, C. Paulsen, J.R. Iglesias, Phys. Rev. Lett. 98, 166406 (2007).

[48] N. Marcano, S.G Magalhaes, B. Coqblin, J.C. Gomez Sal, J.I. Espeso, F.M. Zimmer, J.R. Iglesias, J. Phys. Conf. Series 200, 012111 (2010).

[49] T. Westerkamp, M. Deppe, R. Kucler, M. Brando, C. Geibel, P. Gegenwart, A.P. Pikul, F. Steglich, Phys. Rev. Lett. 102, 206404 (2009).

[50] M. Brando, T. Westerkamp, M. Deppe, P. Gegenwart, C. Geibel, F. Steglich, J. Phys. Conf. Series 200, 012016 (2010).

[51] D. Sherrington, S. Kirkpatrick, Phys. Rev. Lett. 35, 1792 (1975).

[52] Alba Theumann, B. Coqblin, S.G. Magalhaes, A.A. Schmidt, Phys. Rev. B 63, 054409 (2001).

[53] S.G. Magalhaes, A.A. Schmidt, Alba Theumann, B. Coqblin, Eur. Phys. J. B 30, 419 (2002).

[54] S.G. Magalhaes, A.A. Schmidt, F.M. Zimmer, Alba Theumann, B. Coqblin, Eur. Phys. J. B 34, 447 (2003).

[55] D.J. Mattis, Phys. Lett. 56A, 421 (1977).
[56] S.G Magalhaes, F.M. Zimmer, P.R. Krebs, B. Coqblin, Phys. Rev. B 74, 014427 (2006).

[57] J.L. van Hemmen, J. Phys. C: Solid State Phys. 19, L-379 (1986).

[58] F.M. Zimmer, S.G. Magalhaes, B. Coqblin, Physica B 404, 2972 (2009).

[59] S.G. Magalhaes, F.M. Zimmer, B. Coqblin, J. Phys. Conf. Series 273, 012069 (2011).

[60] S. Majumdar, F.V. Sampathkumaran, St. Berger, M. Della Mea, H. Michor, E. Bauer, M. Brando, J. Hemberger, A. Loidl, Solid State Commun. 121, 665 (2002).

[61] S.G. Magalhaes, F.M. Zimmer, B. Coqblin, J. Phys., Condens. Matter 18, 3479 (2006).

[62] R. Vollmer, T. Pietrus, H. v. Lohneysen, R. Chau, M.B. Maple, Phys. Rev. B 61, 1218 (2000).

[63] R.P. Dickey, E.J. Freeman, V.S. Zapf, P.-C. Ho, M.B. Maple, Phys. Rev. B 68, 144402 (2003).

[64] V.S. Zapf, R.P. Dickey, E.J. Freeman, C. Sirvent, M.B. Maple, Phys. Rev. B 65, 024437 (2002).

[65] C.L. Seaman, M.B. Maple, B.W. Lee, S. Ghamaty, M.S. Torikachvili, J.S. Kang, L.Z. Liu, J.W. Allen, D.L. Cox, Phys. Rev. Lett. 67, 2882 (1991).

[66] B. Andraka, A.M. Tsvelik, Phys. Rev. Lett. 67, 2886 (1991).

[67] D. Huo, J. Sakurai, T. Kuwai, Y. Isikawa, Phys. Rev. B 64, 224405 (2001).

[68] O. Tougait, R. Troc, A. Zaleski, H. Noel, Philos. Mag. 87, 1085 (2007).

[69] V.H. Tran, Z. Bukowski, J. Stepien-Damm, A.J. Zaleski, D. Badurski, R. Gorzelniak, Cz. Sulkowski, R. Troc, J. Phys., Condens. Matter 17, 3597 (2005).

[70] M.B. Silva Neto, A.H. Castro Neto, D. Mixson, J.S. Kim, G.R. Stewart, Phys. Rev. Lett. 91, 257206 (2003).

[71] J.A. Mydosh, Spin Glasses: An Experimental Introduction, Taylor and Francis, London 1993.

[72] S.G. Magalhaes, F.M. Zimmer, B. Coqblin, presented at the SCES2011 Conf., Cambridge (England), 2011, to be published in J. Phys., Conf. Series.

[73] J.R. Iglesias, J.I. Espeso, N. Marcano, J.C. Gomez Sal, Phys. Rev. B 79, 195218 (2009).

[74] R.B. Griffiths, Phys. Rev. Lett. 23, 17 (1969).

[75] C. Lacroix, J. Phys. Soc. Jpn. 79, 011008 (2010).

[76] P. Coleman, A.H. Nevidomsky, J. Low Temp. Phys. 161, 182 (2010).

[77] B.S. Shastry, B. Sutherland, Physica B 108, 1069 (1981).

[78] E. Bauer, H. Michor, T. Muramatsu, T. Kanemasa, T. Kagayama, K. Shimizu, Y. Aoki, H. Sato, M. Giovannini, J. Optoelectron. Adv. Mat. 10, 1633 (2008).

[79] K. Sengupta, M.K. Forthaus, H. Kubo, K. Katoh, K. Umeo, T. Takabatake, M.M. Abd-Elmeguid, Phys. Rev. B 81, 125129 (2010).

[80] M.S. Kim, M.C Bennett, M.C. Aronson, Phys. Rev. B 77, 144425 (2008).

[81] P.S. Riseborough, B. Coqblin, S.G Magalhaes, Phys. Rev. B 85, 165116 (2012). 